\title{
HIDMS-PSO with Bio-inspired Fission-Fusion Behaviour and a Quorum Decision Mechanism
}

\author{
Fevzi Tugrul Varna \\ Department of Informatics, University of Sussex \\ Brighton, United Kingdom \\ f.varna@sussex.ac.uk
}

\author{
Phil Husbands \\ Department of Informatics, University of Sussex \\ Brighton, United Kingdom \\ philh@sussex.ac.uk
}

\begin{abstract}
In this study, we propose a new variant of the HIDMS-PSO algorithm with a bio-inspired fission-fusion behaviour and a quorum decision mechanism (FFQ-HIDMS-PSO). In the new algorithm, units are conceptualised as self-organising fission-fusion societies that determine and adopt a suitable behaviour using unit-based quorum decisions. The incorporation of the two bio-inspired mechanisms provide "diversity aware" selforganising units that react to stagnation of particles by adopting a suitable fission-fusion behaviour, leading to a more efficient algorithm capable of maintaining significantly better population diversity throughout the search. The performance of the proposed algorithm was verified with three distinct experiments conducted using CEC'17 and CEC'05 test suites at 30 and 50 dimensions, comparing against 12 state-of-the-art metaheuristics and 12 state-of-the-art PSO variants. The proposed algorithm showed superior performance in these experiments by outperforming all 24 algorithms in all three experiments at 30 and 50 dimensions. The empirical evidence suggests that the proposed method also maintains significantly superior population diversity in comparison to the original HIDMS-PSO.
\end{abstract}

Index Terms - particle swarm optimisation, swarm intelligence, meta-heuristics

\section{INTRODUCTION}

Particle swarm optimisation, proposed in 1995 by Kennedy et al [1] is an optimisation algorithm widely used for a range of problems. Since its invention, due to its simple structure and effectiveness, PSO has attracted a lot of attention from researchers which resulted in many variants [2] and applications in a range of fields [3] [4]. The vast majority of PSO variants proposed in the literature address the problem of premature convergence to improve the performance of the algorithm. The HIDMS-PSO algorithm is a state-of-the-art PSO variant proposed in 2020 by Varna and Husbands [5]. The algorithm performs search using two fixed subpopulations, one homogeneous and one heterogeneous, and an explicit communication model to slow down the loss of population diversity. The intention of this paper is to further improve the depletion of population diversity by redesigning the unit structures in the standard HIDMS-PSO algorithm as selforganising social groups. Self-organising groups are widespread in nature, occurring in species from bacteria colonies to humans. Eusocial animals commonly exhibit self-organising behaviour to resolve various issues in order to survive. In this study, we propose a mechanism composed of a bio-inspired fission-fusion behaviour and a quorum decision mechanism to form self-organised units capable of reacting to improve their diversity. As a result, from this low-level behaviour emerges an overall higher-level population with a significantly improved diversity, which reduced premature convergence and improves search efficiency. Fission-fusion behaviour involves social groups changing their formation over time through either splitting into smaller groups (fission) or merging with other groups (fusion). It is observed in many organisms, including social insects, birds, fish and even humans, as a form of fitness beneficiary mechanism in a social group or colony, used to maximise survival or reproduction, or to minimise the chances of becoming prey. In our algorithmic model, fission-fusion behaviour is employed as a reactive mechanism through the creation of "diversity aware" units that exhibit fission-fusion behaviour when a unit's diversity exhibits a downward trend. Many colony and social group based species, such as ants and honeybees, make group decisions, and in our behavioural model units make a group decision on when to adopt fissionfusion behaviour, based on a quorum response. As a result, these two incorporated mechanisms provide units with the ability to self-organise by reaching a decision and adopting a behaviour through consensus, which in turn significantly improves population diversity and the overall performance of the algorithm.

\section{BACKGROUND}

This section provides the necessary background information about the canonical PSO and HIDMS-PSO.

\section{A. Particle Swarm Optimisation}

Agents in the PSO algorithm mimics the social movements of birds flocking. The algorithm initiates the search with $N$ randomly defined particles that represents a solution in the search space. Each particle has a velocity, position and personal best position at time step $t$ represented by notations $\vec{v}_{i}^{(t)}, \vec{x}_{i}^{(t)}$ and pbest, respectively. At every iteration, particles update their velocity and position using the following two equations until a termination criterion is met:

$$
\vec{v}_{i}^{(t+1)}=\omega \vec{v}_{i}^{(t)}+c_{1} \vec{r}_{1}\left(p \overrightarrow{b e s t} t_{i}-\vec{x}_{i}^{(t)}\right)+c_{2} \vec{r}_{2}\left(g b \vec{e} s t-\vec{x}_{i}^{(t)}\right)
$$




$$
\vec{x}_{i}^{(t+1)}=\vec{x}_{i}^{(t)}+\vec{v}_{i}^{(t)}
$$

Where $\omega$ is the inertia weight used to control the impact of the previous velocity, constants $c_{1}$ and $c_{2}$ control the attraction rate/level for cognitive (pbest) and social (gbest) attraction, $\vec{r}_{1}$ and $\vec{r}_{2}$ are random vectors $\in[0,1]^{n}$.

\section{B. HIDMS-PSO}

The HIDMS-PSO algorithm introduced a new master-slave inspired dynamic topological structure with homogeneous and heterogeneous subpopulations and two movement strategies, namely, inward and outward-oriented strategies. The small subswarm entities in the HIDMS-PSO algorithm are called units and each unit constitutes a single master particle and 3 slave particles with distinct types. Master and slave particles retain their roles throughout the search process. Distinguishing the slave particles by assigning unique types enables particles to behave heterogeneously. In addition, through the types of slaves, communication among particles are significantly confined to avoid fast positional information sharing to avert loss of diversity. Figure 1 displays the visual depiction of the unit topological structure.

Information flow and the way particles interact with one another has an immense impact on the population diversity and particles' guidance, hence the overall search process. The HIDMS-PSO algorithm employs a communication model to control the flow of information and the interaction between particles. The communication model restricts information flow and allows particles to exchange information through masterto-master and slave-to-slave communication (see Fig. 2). The main communication is governed by the following rules:

1) The responsibility of unit-to-unit communication is assigned to particles with slave roles. Hence, only slave particles can communicate with other slaves in different units, and unit-to-unit communication is formed in this way. However, slave particles in the same unit cannot communicate with each other as each slave have a different type.

2) Master particles are prohibited from external communication (with other units). Hence, master particles can only communicate internally with their own slave particles.

a) Search Behaviour: In the HIDMS-PSO algorithm, the initial population is divided into two equal subpopulations, one homogeneous and one heterogeneous, and each subpopulation adopts a distinct movement strategy (Fig. 3). The homogeneous subpopulation uses the update equation of the canonical PSO algorithm, whereas the heterogeneous subpopulation is used to form $N$ unit structures and adopts inward and outward-oriented strategies. Both movement strategies used in the HIDMS-PSO have a distinct purpose. The inwardoriented movement directs particles to a potentially better position in the search space using the information from its own

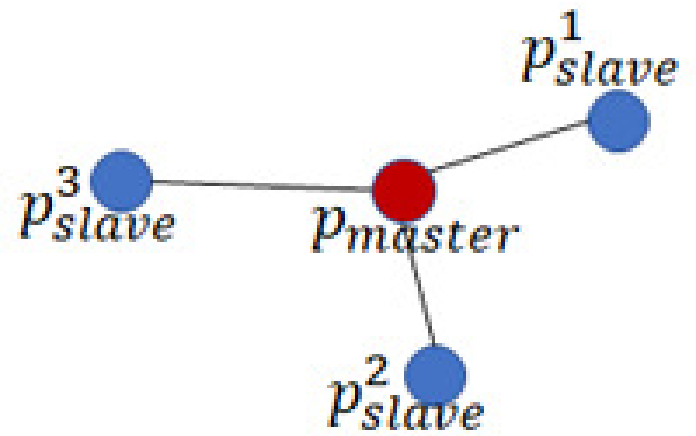

Fig. 1. Topological structure of a single unit.

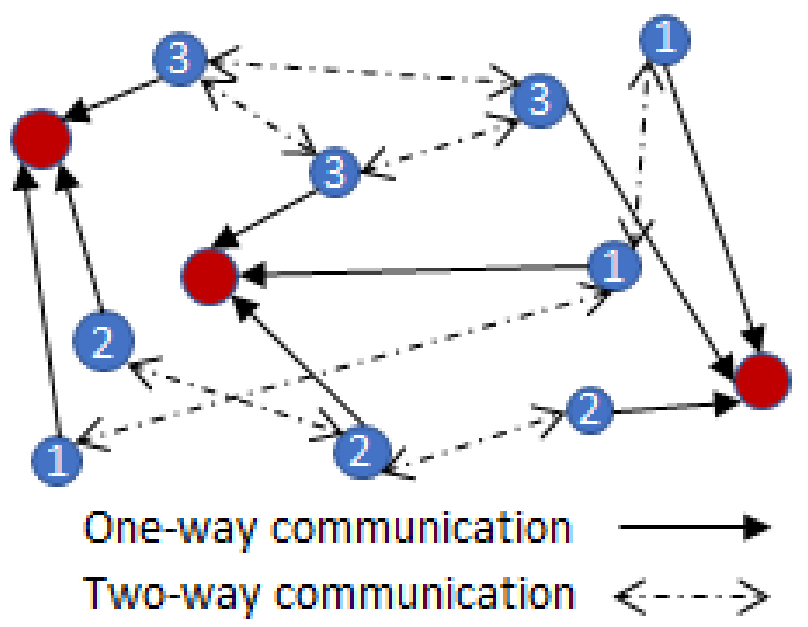

Fig. 2. The visual depiction of the communication model between 3 units.

unit members. On the contrary, the latter approach, outwardoriented movement, influence particles to move in the direction of members of other units.

b) Inward-oriented strategy: As briefly mentioned, the inward-oriented movement exploits the unit's internal information to provide distinct guidance to members with different roles (e.g. master and slave). The master particle randomly selects and uses either equation from 3-5 to update its velocity for this movement scheme.

$$
\vec{v}_{m}^{(t+1)}=\omega^{(t)} \vec{v}_{m}^{(t)}+c_{1} \vec{r}_{1}\left(p b \overrightarrow{b e s} t_{m}-\vec{x}_{m}^{(t)}\right)+c_{2} \vec{r}_{2}\left(\vec{x}_{s}^{d i s}-\vec{x}_{m}^{(t)}\right)
$$

Where $\vec{v}_{m}^{(t)}$ is the velocity, pbest $\vec{m}_{m}$ is the personal best position, $\vec{x}_{m}^{(t)}$ is the position of the master particle at time $t$ and, $\vec{x}_{s}^{d i s}$ is the particle with the lowest positional similarity in the $N^{t h}$ unit. The purpose of guiding in the direction of the least similar particle's position is to prevent the stagnation of the master particle, as the master particle has a significant influence on other members of the unit.

$\vec{v}_{m}^{(t+1)}=\omega^{(t)} \vec{v}_{m}^{(t)}+c_{1} \vec{r}_{1}\left(\right.$ pbest $\left._{m}-\vec{x}_{m}^{(t)}\right)+c_{2} \vec{r}_{2}\left(\vec{x}_{s}^{\text {best }}-\vec{x}_{m}^{(t)}\right)$ 


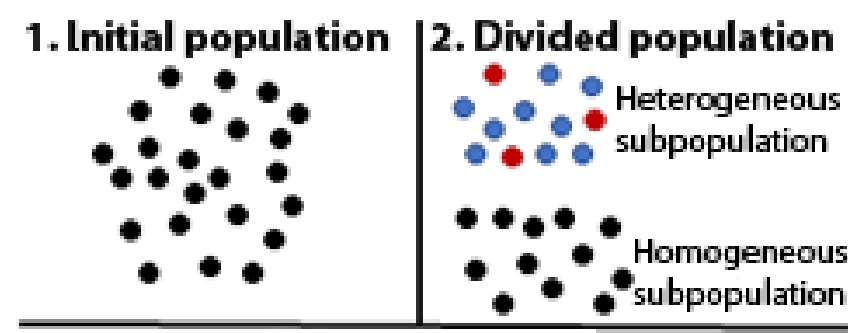

3. Search Process

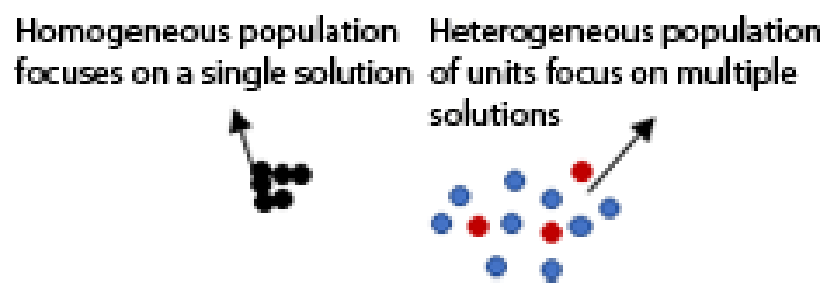

Fig. 3. Search phases of the HIDMS-PSO algorithm.

Where $\vec{x}_{s}^{b e s t}$ is the $N^{\text {th }}$ unit's fittest slave particle at time $t$. The master particle's movement towards the fittest slave encourages local exploration.

$\vec{v}_{m}^{(t+1)}=\omega^{(t)} \vec{v}_{m}^{(t)}+c_{1} \vec{r}_{1}\left(\right.$ pbest $\left._{m}-\vec{x}_{m}^{(t)}\right)+c_{2} \vec{r}_{2}\left(\vec{x}_{s}^{a v g}-\vec{x}_{m}^{(t)}\right)$

Where $\vec{x}_{s}^{a v g}$ is the average position of all slaves within the master's current unit. On the contrary, for the slave particles, the only option provided for this strategy is to move towards the unit master and personal best position of the slave particle, as shown in Eq. 6.

$$
\vec{v}_{s}^{(t+1)}=\omega^{(t)} \vec{v}_{i}^{(t)}+c_{1} \vec{r}_{1}\left(p b \vec{e} s t_{s}-\vec{x}_{s}^{(t)}\right)+c_{2} \vec{r}_{2}\left(\vec{x}_{m}-\vec{x}_{s}^{(t)}\right)
$$

Where $\vec{v}_{s}^{(t)}$ is the velocity, pbest $\vec{s}_{s}$ is the personal best position, $\vec{x}_{s}$ is the position of the slave particle and, $\vec{x}_{m}$ is the position of master particle of the $N^{t h}$ unit.

c) Outward-oriented strategy: As opposed to the inwardoriented strategy, the outward-oriented movement enables particles to learn from other units while maintaining their hierarchical master-slave structure. The velocity of the master particle is updated by randomly selecting either of the following equations (7-9):

$\vec{v}_{m}^{(t+1)}=\omega^{(t)} \vec{v}_{m}^{(t)}+c_{1} \vec{r}_{1}\left(p b \overrightarrow{e s t_{m}}-\vec{x}_{m}^{(t)}\right)+c_{2} \vec{r}_{2}\left(\vec{x}_{u n i t}^{a v g}-\vec{x}_{m}^{(t)}\right)$

Where $\vec{v}_{m}^{(t)}$ is the velocity, pbest $\vec{m}_{m}$ is the personal best position, $\vec{x}_{m}^{(t)}$ is the position of the master particle at time $t$ and, $\vec{x}_{\text {unit }}^{\text {avg }}$ is the mean position of particles in the $N^{t h}$ unit.

$\vec{v}_{m}^{(t+1)}=\omega^{(t)} \vec{v}_{m}^{(t)}+c_{1} \vec{r}_{1}\left(p \overrightarrow{b e s} t_{m}-\vec{x}_{m}^{(t)}+c_{2} \vec{r}_{2}\left(\vec{x}_{u n i t}^{m}-\vec{x}_{m}^{(t)}\right)\right.$

Where $\vec{x}_{u n i t}^{m}$ is the master particle's position from a randomly chosen unit. $\vec{v}_{m}^{(t+1)}=\omega^{(t)} \vec{v}_{m}^{(t)}+c_{1} \vec{r}_{1}\left(\vec{x}^{a v g}-\vec{x}_{m}^{(t)}\right)+c_{2} \vec{r}_{2}\left(\vec{x}_{u n i t}^{m}-\vec{x}_{m}^{(t)}\right)$

Where $\vec{x}^{a v g}$ is the mean position of unit members the particle belongs to and $\vec{x}_{u n i t}^{m}$ is the randomly selected master particle's position from any unit. The slave particles that adopt the outward-oriented movement strategy are guided towards another slave particle (of the same type) from a randomly selected unit using the following velocity update equation:

$$
\vec{v}_{s}^{(t+1)}=\omega^{(t)} \vec{v}_{s}^{(t)}+c_{1} \vec{r}_{1}\left(\text { pbest } t_{s}-\vec{x}_{s}^{(t)}\right)+c_{2} \vec{r}_{2}\left(\vec{x}_{\text {unit }}^{\text {rnd }}-\vec{x}_{s}^{(t)}\right)
$$

Where $\vec{v}_{s}^{(t)}$ is the velocity, pbest $\vec{s}_{s}$ is the personal best position, $\vec{x}_{s}$ is the slave particle's position and, $\vec{x}_{u n i t}^{\text {rnd }}$ is the position of a randomly selected same type of slave in any unit.

The HIDMS-PSO algorithm combines both homogeneous and heterogeneous subpopulations to balance the exploration and exploitation during the search process. In addition, the previously mentioned two movement strategies provide the necessary scope of movement for particles to maintain diversity and potentially avoid local optima [5].

\section{The Proposed Algorithm}

As oppose to the standard HIDMS-PSO, in the proposed algorithm (FFQ-HIDMS-PSO) the search process initiates with a single population of $n$ units of the type shown in Fig 1. Each unit's diversity $\left(\delta_{n}\right)$ is calculated at specific intervals defined by $\frac{F F_{\text {period }}}{10}$, where $F F_{\text {period }}$ is the period of time particles are allowed to adopt the fission-fusion behaviour. $F F_{\text {period }}$ may range from $1-10 \%$ of the maximum number of iterations. After this period, fissioned/fused units are randomly reformed as units while retaining their current particle positions. The $\delta_{n}$ is only calculated for units that have not undergone fissionfusion behaviour and is used as a threshold to initiate the fission-fusion behaviour. The $\delta_{n}$ is calculated as

$$
\delta_{n}=\sum_{j=1}^{N} M S E\left(\overrightarrow{x_{m}}, \overrightarrow{x_{j}}\right)
$$

Where MSE is the mean square error, $\overrightarrow{x_{m}}$ is the position of the master particle of the $n^{t h}$ unit, $\overrightarrow{x_{j}}$ is the jth slave particle of the $n^{\text {th }}$ unit and $N$ is the number of slave particles. if the $n^{t h}$ unit's $\delta$ is less than the average $\delta$, the unit qualifies for fissionfusion behaviour. The type of behaviour (fission or fusion) is randomly selected and the behaviour is only adopted after a group decision. The group decision is based on the quorum response of each member of the unit and it is calculated as

$$
Q R_{j}=\frac{M S E\left(\vec{M}, \overrightarrow{S_{j}}\right)}{1+\left(\frac{\alpha_{j}}{\beta}\right)^{\gamma / 10}}
$$

Where $Q R_{j}$ is the quorum response of the $j^{\text {th }}$ member, $\vec{M}$ is the position of the unit master, and $\overrightarrow{S_{j}}$ is the position of the $j^{\text {th }}$ slave, $\alpha$ is the number of fissioned/fused conspecific particle at time $t, \beta$ is the total number of particles with fission/fusion behaviour and $\gamma$ is the fitness rank of the $j^{\text {th }}$ 


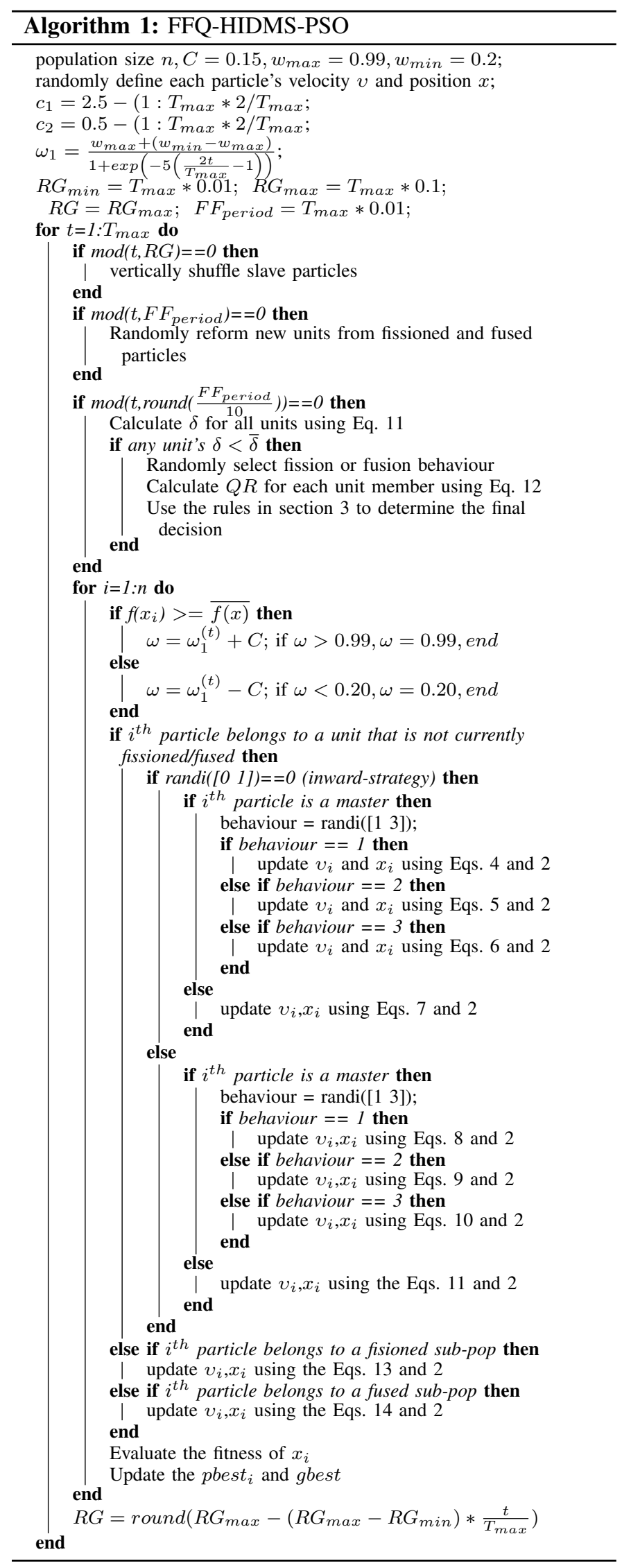

\section{Fissioned Unit Fused Unit}
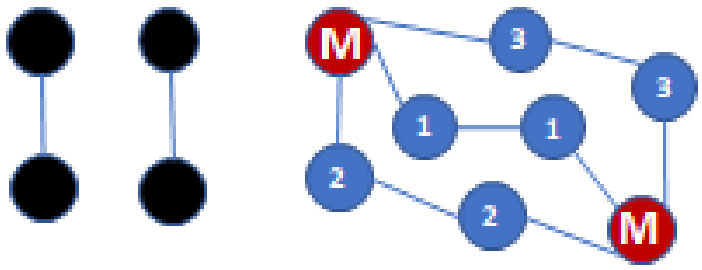

Fig. 4. Visual depictions of fissioned and fused units.

member ( 1 to 4 , the fitter the particle, the higher the rank). Eq. 12 is an adapted version of the equation used to model animal group behavior in [6], and it allows members of a unit to individually gather information and compare findings with other unit members to reach the final decision. The $\mathrm{QR}$ equation essentially mimics how decentralised animal groups without a "leader" form a consensus. The decision is finalised by counting the number of unit members with $Q R_{j}>\overline{Q R}$, where $\overline{Q R}$ is the average quorum response for the unit. Subsequently, the following rules are applied to determine the final decision

1) If more than 2 unit members have greater $Q R$ values than $\overline{Q R}$, fission or fusion behaviour is adopted.

2) If more than 2 unit members have lower $Q R$ values than $\overline{Q R}$, fission or fusion is not adopted.

3 ) If the number of unit members with $\mathrm{QR}$ values greater and less than $\overline{Q R}$ are the same or if all $\mathrm{QR}$ values of unit members are the same, we use the $\mathrm{QR}$ value of the unit master in place of $\overline{Q R}$ and proceed according to the first two rules.

As mentioned previously, many animals exhibit fissionfusion behaviour by temporarily splitting up and merging hence, fission-fusion behaviour is a strategy employed by social animals to reorganise their groups to increase or reduce potential loss of fitness. Fig 4 shows the visual depictions of a unit with fission and fusion behaviour.

\section{A. Fission-Fusion Behaviour}

As briefly mentioned, in this study, we conceptualise each unit as a fission-fusion society like those that exist in nature. Fission-fusion societies split up into smaller social groups or merge to form larger groups. This type of behaviour is usually adopted after a group decision, if it is the optimal option for all individuals to behave in smaller or larger social units to, for instance, forage, mate or exhibit predatory behaviour. In our behavioural model, units that adopt fission behaviour are divided into two equal sub-units and as oppose to the unit structure, particles within these sub-units do not have masterslave roles. The units that have not adopted fission-fusion behaviour remain as part of the heterogeneous unit population and control their movements according to the original HIDMSPSO rules (Sect. III, and see the pseudocode). The fission 
behaviour provides four different exemplars to guide particles and their velocities are calculated using the following equation:

$\vec{v}_{i}^{(t+1)}=\omega^{(t)} \vec{v}_{i}^{(t)}+c_{1} \vec{r}_{1}\left(p b \overrightarrow{e s} t_{i}-\vec{x}_{i}^{(t)}\right)+c_{2} \vec{r}_{2}\left(\vec{x}_{\text {fission }}-\vec{x}_{i}^{(t)}\right)$

Where $\vec{x}_{\text {fission }}$ is the position of a particle randomly selected from: the most diverse fissioned sub-unit (diversity of a sub-unit is based on the positional similarity and can be calculated using the MSE function), a random fissioned sub-unit, the first fissioned and the last fissioned sub-units. As oppose to the fission behaviour, the fusion behaviour combines a maximum of two units while maintaining master-slave roles (as shown in Fig 4). The fusion behaviour allows the fused units to influence each other at individual level resulting in a potentially more diverse units at higher level. The particles within fused units use the following equation to update their velocity

$\vec{v}_{i}^{(t+1)}=\omega^{(t)} \vec{v}_{i}^{(t)}+c_{1} \vec{r}_{1}\left(\right.$ pbest $\left._{i}-\vec{x}_{i}^{(t)}\right)+c_{2} \vec{r}_{2}\left(\vec{x}_{\text {fusion }}-\vec{x}_{i}^{(t)}\right)$

Where $\vec{x}_{\text {fusion }}$ is randomly chosen as either the position of the conspecific particle (e.g. slave type) within the unit, the most diverse particle relative to its master within the fused unit, or the particle's personal best position.

By combining both behaviours, units regain diversity, or at least significantly slow down loss of diversity. This progressively extends to the overall population, resulting in particles escaping from local optima. Since fission-fusion behaviour is triggered as a result of loss of diversity in a unit, in both behaviours, the motivation is to guide particles using different sources, as defined in Eqs. 13 and 14, to avoid stagnation and potentially improve diversity of each individual to prepare them to reunite and form new units with sufficient diversity to carry out the search.

The FFQ-HIDMS-PSO algorithm uses the same parametric settings as the standard HIDMS-PSO; for a detailed description of the parameters, refer to the original study [5]. Besides the standard PSO parameters $c_{1}, c_{2}$ and $\omega$, the HIDMS-PSO employ an additional parameter $R G$ to reshape unit structures periodically (see the pseudocode).

\section{EXPERIMENTAL RESUlts AND Discussions}

This section discusses the experimental setup and the corresponding results. In the first subsection, we detail the experimental design and statistical analysis of the results. In the second subsection, we discuss the experimental results in detail.

\section{A. Experimental Setup}

This study carried out three distinct experiments to assess and verify the FFQ-HIDMS-PSO algorithm's performance on the CEC'05 and CEC'17 benchmark test suites against various well-known baseline metaheuristics and state-of-theart PSO variants. In the first experiment, the performance of the proposed algorithm is tested using the CEC'17 test suite. The CEC'17 test suite consists of 30 and CEC'05 consists of 25 continuous optimisation test functions. For the first and second experiments, we replicated the experiments conducted in [5] and for the third experiment, study [27] (which uses a different set of comparator algorithms) was replicated to produce comparable results. The results of the proposed algorithm is compared with 11 state-of-the-art evolutionary methods including two canonical PSO algorithms with different parameter values $\left(\omega=0.9 \rightarrow 0.4, c_{1}, c_{2}=2\right.$ and $\omega=0.4, c_{1}, c_{2}=2$ ), and evolutionary algorithms (including the bat algorithm (BA) [7], grey wolf optimiser (GWO) [8], butterfly optimisation algorithm (BOA) [9], whale optimisation algorithm (WOA) [10], moth flame optimisation (MFO) [11], artificial bee colony (ABC) [12], invasive weed optimisation (IWO) [13], flower pollination algorithm (FPA) [14] and cuckoo search algorithm (CS) $(\mathrm{p}=0.25)$ [15]. In both the second and third experiments, the proposed algorithm's performance was tested using the CEC' 05 test suite and results were compared with a total of 12 state-of-the-art PSO variants including $\chi \mathrm{PSO}$ (ring with neighborhood radius $n_{r}=2, \phi=$ $\left.4.1, \chi=0: 72984, c_{1}, c_{2}=2.05\right)$ [16], BBPSO [17], DMSPSO $\left(\omega=0.729, c_{1}, c_{2}=1.49445, V_{\max }=0.5 *\right.$ Range $)$ [18], FIPS [19], UPSO [20], CLPSO $\left(\omega=0.9 \rightarrow 0.2, c_{1}, c_{2}=\right.$ $1.49445, V_{\max }=0.2 *$ Range $)$ [21], HIDMS-PSO $(\omega=$ $\left.0.99 \rightarrow 0.29, c_{1}=2.5 \rightarrow 0.5, c_{2}=0.5 \rightarrow 2.5\right)$ [5], HPSOTVAC [22], FDR-PSO [23], HCLDMS-PSO $(\omega=0.99 \rightarrow$ $0.29, c_{1}=2.5 \rightarrow 0.5, c_{2}=0.5 \rightarrow 2.5, \mathrm{pm}=0.1, V_{\max }=$ $0.5 *$ Range)) [24], HCLPSO [25] and MNHPSO-JTAC [26]. In the first experiment, the population size was set to 100 for all metaheuristics, and 40 for the two PSO algorithms and the FFQ-HIDMS-PSO. In the second and third experiments, the comparison algorithms comprise only the PSO variants. For all PSO variants, the size of the population was set as 40 [5]. The problems used in the first two experiments were tested 30 times and in the third experiment 100 times for 300,000 and 500,000 functions evaluations at 30 and 50 dimensions, respectively. Studies [5] [27] and the original papers can be referred for detailed parametric settings used for the comparison algorithms. The mean errors are recorded for each problem and the results are shown in Tables I-VI. The average and final ranks obtained from the mean performances in all three experiments are displayed in Table VII-IX. The Wilcoxon signed-rank test is applied to measure the statistical significance of the differences between FFQ-HIDMS-PSO and the comparison algorithms. The obtained results for the first experiment specify that result is significant for all comparison algorithms for 30 and 50-dimensional problems. For the second experiment conducted, the result is significant for all comparison algorithms apart from HCLDMS-PSO and DMSPSO. For 50-dimensional problems, the result is not significant for only HIDMS-PSO and significant for the rest of the compared methods. Lastly, the result of the third experiment showed that for 30-dimensional problems, the result is only not significant for CLPSO and BBPSO and for 50 dimensional problems, the result is significant for all comparison algorithm employed in the experiment at $p<0.05$. Due to the length 
restrictions of this paper, experimental results are partially included. External supplementary material is provided for complete results of experiments that can be accessed from users.sussex.ac.uk/fv47/FFQ-HIDMS-PSO.pdf.

\section{B. Experiment Results}

The results for the first experiment conducted on the CEC' 17 test suite at 30 dimensions show that the proposed algorithm (FFQ-HIDMS-PSO) outperformed comparison methods for problems F5, F7, F8, F9, F11, F12, F16, F17, F20, F21, F22, F23, F24,F27, F29 and F30. The HIDMS-PSO algorithm achieved the best mean performance for problems F3 and F28. CS outperformed comparison algorithms for problems F14, F14, F15, F18 and F19. For problems F1, F4, F6, F10, $\mathrm{F} 25$ and $\mathrm{F} 26, \mathrm{ABC}$ attained the best mean performance. BA, GWO, BOA, WOA, MFO, FPA, IWO, $\mathrm{PSO}_{1}$ and $\mathrm{PSO}_{2}$ did not outperform any of the comparison algorithms on any problems at 30 dimensions. The second experiment conducted on the CEC'17 test suite for the problem size of 50 dimensions reveals that FFQ-HIDMS-PSO attained the best mean performance for problems F1, F5, F7, F8, F9, F10, F11, F12, F13, F16, F17, F20, F21, F22, F23, F24, F25, F26, F29 and F30. For problems F3, F4 and F6, HIDMS-PSO achieved the best mean performance. The CS algorithm outperformed the comparison methods for problems F14, F15, F18, F19 and F28. Lastly, BA, GWO, BOA, WOA, MFO, FPA, IWO, $\mathrm{PSO}_{1}$ and $\mathrm{PSO}_{2}$ did not outperform any of the comparison algorithms on any problems at 50 dimensions. The results for the second experiment conducted on the CEC'05 test suite at 30 dimensions reveal that FFQ-HIDMS-PSO outperformed comparison state-of-theart PSO variants for problems F5, F6, F10, F14, F19, F22, and F25. HCLDMS-PSO attained the best mean performance for problems F6, F17, F18, F20, F21 and F24. For problems F2, F7, F8, F11, F12 and F23, the HIDMS-PSO algorithm outperformed the comparison methods. HCLPSO achieved the best performance for problems F9, F13 and F15. MNHPSO-JTVAC outperformed comparison methods for a single problem (F3) while HPSO-TVAC attained the best performance for F1 and F16. The same experiment conducted at 50 dimensions reveal that the proposed algorithm outperformed the comparison methods for problems F4, F5, F10, F14, F16, F18, F19, F20, F21, F22, F24, and F25. The HIDMS-PSO algorithm attained the best mean performance for problems F2, F6, F7, F8, F11, F12 and F23. For problems F9, F13, F15 and F17, the HCLPSO algorithm outperformed the comparison methods. HPSO-TVAC and MNHPSO-JTAC each achieved the best performance for a single problem: F1 and F3, respectively. HCLDMS-PSO and FDR-PSO did not outperform any of the algorithms for any problems at 50 dimensions. The results for the third experiment conducted on the CEC'05 test suite at 30 dimensions reveal that FFQ-HIDMS-PSO attained the best mean performance for problems F4, F5, F10, F11, F14, F17, F19, F20, F22 and F25. CLPSO outperformed comparison methods for problems F1, F6, F8, F9, F13, F15, F18, F21 and F23. For problems F1, F2, F3, F12 and F16, CLPSO obtained the best performance and DMSPSO achieved the best mean performance for a single problem of $\mathrm{F} 7 . \chi \mathrm{PSO}, \mathrm{BBPSO}$ and CLPSO attained an equal performance for problem F24. FIPS and UPSO did not outperform any of the algorithms for any problems at 30 dimensions. The same experiment conducted at 50 dimensions reveal that FFQ-HIDMS-PSO achieved the best performance for problems F4, F5, F10, F16, F17, F18, F19, F20 and F25. The CLPSO algorithm attained the best performance for problems F1, F9, F15, F21, F23 and F24. For problems F1, F2, F3, F6, F12, and F23, BBPSO achieved the best mean performance, UPSO outperformed the comparison algorithms for problems F8, F11, F13 and F14 and DMSPSO outperformed the comparison algorithms in a single case for problem F7. FIPS and $\chi$ PSO did not outperform any of the algorithms for any problems at 50 dimensions. An additional experiment was conducted to observe and compare the rate of population diversity for the standard HIDMSPSO algorithm and the new proposed variant. Fig 5 shows average value of population diversity over 20 consecutive runs for both algorithms. The empirical evidence clearly indicates that the proposed algorithm, FFQ-HIDMS-PSO, is capable of avoiding the depletion of population diversity. It is also worth noting that, in addition to a communication model mentioned in previous sections, HIDMS-PSO employs a nonuniform mutation operator at each iteration which significantly contributes to evading stagnation [5]. However, both of those mechanisms are not included in FFQ-HIDMS-PSO indicating the effectiveness of the mechanisms proposed in this study.

\section{CONCLUSIONS}

The present study proposed an extension of the state-ofthe-art HIDMS-PSO algorithm that incorporates bio-inspired fission-fusion behaviour and a quorum decision mechanism, FFQ-HIDMS-PSO. The original algorithm was trimmed down by discarding the mutation operator and the communication model that was proposed in the original study. The new algorithm was equipped with bio-inspired fission-fusion behaviour and the quorum decision mechanisms provide the new algorithm with "diversity aware" units capable of adopting a suitable behaviour through quorum decision to regain a unit's diversity and boost the overall population diversity. The empirical evidence suggests that the new algorithm is superior in maintaining the population diversity throughout the search in comparison to the original HIDMS-PSO algorithm. The proposed algorithm was tested with three distinct experiments on the CEC'17 and CEC'05 test suites at 30 and 50 dimensions against 12 state-of-the-art metaheuristics and 12 stateof-the-art PSO variants. The proposed algorithm has shown a superior performance by outperforming all 24 algorithms in all conducted experiments. The present can be extended by further improving or applying the proposed algorithm to practical engineering problems.

\section{REFERENCES}

[1] R. C. Eberhart, and J. Kennedy, "A new optimizer using particle swarm theory", Proc. of the Sixth Int. Symposium on Micromachine and Human Science, Nagoya, Japan. pp. 39-43, 1995 

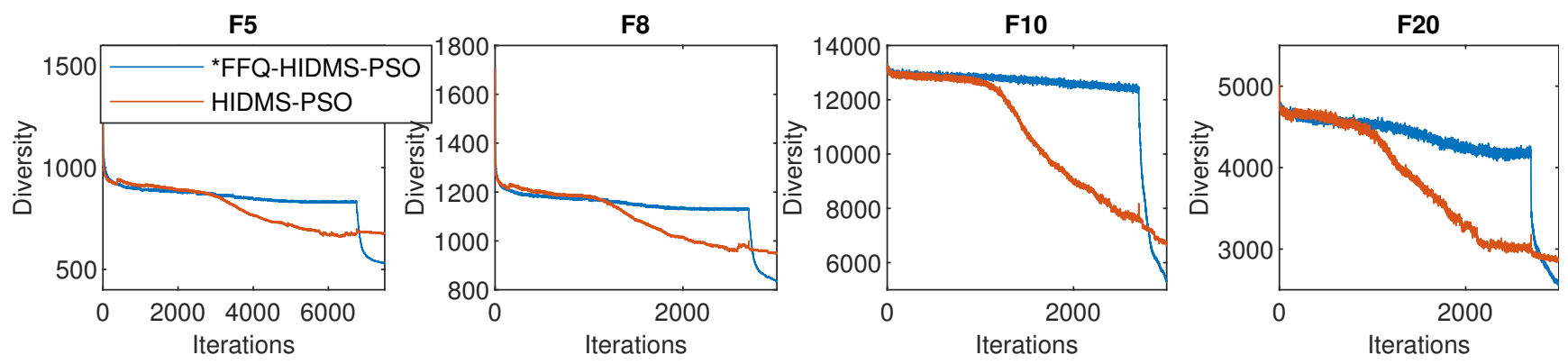

Fig. 5. Comparison of population diversity for HIDMS-PSO and FFQ-HIDMS-PSO for CEC'17 test suite problems F5, F8, F10 and F20.

TABLE I

THE RESULTS OBTAINED FOR THE FIRST EXPERIMENT CONDUCTED USING THE CEC'17 TEST SUITE FOR PROBLEM SIZE OF 30 DIMENSIONS.

\begin{tabular}{|c|c|c|c|c|c|c|c|c|c|}
\hline & F1 & F3 & F4 & F5 & F6 & F7 & F8 & F9 & F10 \\
\hline BA & $7.3 \mathrm{E}+10$ & $2.2 \mathrm{E}+05$ & $2.1 \mathrm{E}+04$ & $5.1 \mathrm{E}+02$ & $1.1 \mathrm{E}+02$ & $1.5 \mathrm{E}+03$ & $4.3 \mathrm{E}+02$ & $2.1 \mathrm{E}+04$ & $8.8 \mathrm{E}+03$ \\
\hline GWO & $1.1 \mathrm{E}+09$ & $2.9 \mathrm{E}+04$ & $1.5 \mathrm{E}+02$ & $8.7 \mathrm{E}+01$ & $4.0 \mathrm{E}+00$ & $1.6 \mathrm{E}+02$ & $7.7 \mathrm{E}+01$ & $5.4 \mathrm{E}+02$ & $2.8 \mathrm{E}+03$ \\
\hline BOA & $3.0 \mathrm{E}+10$ & $6.7 \mathrm{E}+04$ & $2.5 \mathrm{E}+03$ & $3.3 \mathrm{E}+02$ & $6.4 \mathrm{E}+01$ & $5.1 \mathrm{E}+02$ & $2.9 \mathrm{E}+02$ & $6.9 \mathrm{E}+03$ & $7.7 \mathrm{E}+03$ \\
\hline WOA & 2.1E+06 & $1.6 \mathrm{E}+05$ & $1.5 \mathrm{E}+02$ & $2.7 \mathrm{E}+02$ & $6.6 \mathrm{E}+01$ & $5.1 \mathrm{E}+02$ & $1.9 \mathrm{E}+02$ & $7.7 \mathrm{E}+03$ & $4.8 \mathrm{E}+03$ \\
\hline MFO & $8.1 \mathrm{E}+09$ & $7.7 \mathrm{E}+04$ & $5.1 \mathrm{E}+02$ & $1.8 \mathrm{E}+02$ & $2.5 \mathrm{E}+01$ & $3.5 \mathrm{E}+02$ & $1.7 \mathrm{E}+02$ & $5.1 \mathrm{E}+03$ & 4.1E+03 \\
\hline $\mathrm{ABC}$ & $1.3 \mathrm{E}+02$ & $1.2 \mathrm{E}+05$ & $3.4 \mathrm{E}+01$ & $8.8 \mathrm{E}+01$ & $0.0 \mathrm{E}+00$ & $1.0 \mathrm{E}+02$ & $8.9 \mathrm{E}+01$ & $8.2 \mathrm{E}+02$ & $2.3 \mathrm{E}+03$ \\
\hline FPA & $1.1 \mathrm{E}+11$ & $1.8 \mathrm{E}+06$ & $3.6 \mathrm{E}+04$ & $6.2 \mathrm{E}+02$ & $1.3 \mathrm{E}+02$ & $2.5 \mathrm{E}+03$ & $5.6 \mathrm{E}+02$ & $3.1 \mathrm{E}+04$ & $9.1 \mathrm{E}+03$ \\
\hline $\mathrm{CS}$ & $1.9 \mathrm{E}+04$ & $4.5 \mathrm{E}+04$ & $7.5 \mathrm{E}+01$ & $1.4 \mathrm{E}+02$ & $5.0 \mathrm{E}+01$ & $1.6 \mathrm{E}+02$ & $1.3 \mathrm{E}+02$ & $4.6 \mathrm{E}+03$ & $3.7 \mathrm{E}+03$ \\
\hline IWO & $3.0 \mathrm{E}+03$ & $6.4 \mathrm{E}+03$ & $8.8 \mathrm{E}+01$ & $4.1 \mathrm{E}+02$ & $7.2 \mathrm{E}+01$ & $2.0 \mathrm{E}+03$ & $3.5 \mathrm{E}+02$ & $7.6 \mathrm{E}+03$ & $4.7 \mathrm{E}+03$ \\
\hline $\mathrm{PSO}_{1}$ & $1.3 \mathrm{E}+11$ & $3.9 \mathrm{E}+08$ & 4.4E+04 & $6.8 \mathrm{E}+02$ & $1.4 \mathrm{E}+02$ & $2.7 \mathrm{E}+03$ & $6.1 \mathrm{E}+02$ & $3.8 \mathrm{E}+04$ & $9.6 \mathrm{E}+03$ \\
\hline $\mathrm{PSO}_{2}$ & $1.3 \mathrm{E}+11$ & $3.9 \mathrm{E}+08$ & 4. $4 \mathrm{E}+04$ & $6.8 \mathrm{E}+02$ & $1.4 \mathrm{E}+02$ & $2.7 \mathrm{E}+03$ & $6.1 \mathrm{E}+02$ & $3.8 \mathrm{E}+04$ & $9.6 \mathrm{E}+03$ \\
\hline HIDMS-PSO & $2.6 \mathrm{E}+03$ & $2.5 \mathrm{E}-10$ & $6.2 \mathrm{E}+01$ & $5.3 \mathrm{E}+01$ & $9.6 \mathrm{E}-03$ & $9.2 \mathrm{E}+01$ & $5.2 \mathrm{E}+01$ & $3.6 \mathrm{E}+00$ & $2.9 \mathrm{E}+03$ \\
\hline${ }^{*}$ FFQ-HIDMS-PSO & $1.8 \mathrm{E}+03$ & $1.0 \mathrm{E}+01$ & $8.9 \mathrm{E}+01$ & $2.6 \mathrm{E}+01$ & $2.1 \mathrm{E}-01$ & $6.6 \mathrm{E}+01$ & $2.5 \mathrm{E}+01$ & $1.1 \mathrm{E}-01$ & $3.1 \mathrm{E}+03$ \\
\hline
\end{tabular}

TABLE II

THE RESULTS OBTAINED FOR THE FIRST EXPERIMENT CONDUCTED USING THE CEC' 17 TEST SUITE FOR PROBLEM SIZE OF 50 DIMENSIONS.

\begin{tabular}{|c|c|c|c|c|c|c|c|c|c|}
\hline & F1 & F3 & F4 & F5 & F6 & F7 & F8 & F9 & F10 \\
\hline$\overline{\mathrm{BA}}$ & $1.7 \mathrm{E}+11$ & $8.2 \mathrm{E}+07$ & $6.3 \mathrm{E}+04$ & $9.5 \mathrm{E}+02$ & $1.3 \mathrm{E}+02$ & $3.3 \mathrm{E}+03$ & $9.7 \mathrm{E}+02$ & $7.5 \mathrm{E}+04$ & $1.6 \mathrm{E}+04$ \\
\hline GWO & $4.6 \mathrm{E}+09$ & $7.0 \mathrm{E}+04$ & $4.3 \mathrm{E}+02$ & $1.7 \mathrm{E}+02$ & $1.1 \mathrm{E}+01$ & $3.0 \mathrm{E}+02$ & $2.0 \mathrm{E}+02$ & $3.7 \mathrm{E}+03$ & $5.6 \mathrm{E}+03$ \\
\hline $\mathrm{BOA}$ & $4.3 \mathrm{E}+10$ & $2.2 \mathrm{E}+05$ & $9.9 \mathrm{E}+03$ & $6.2 \mathrm{E}+02$ & $7.9 \mathrm{E}+01$ & $1.1 \mathrm{E}+03$ & $6.5 \mathrm{E}+02$ & $2.8 \mathrm{E}+04$ & $1.4 \mathrm{E}+04$ \\
\hline WOA & 7.1E+06 & $7.8 \mathrm{E}+04$ & $2.8 \mathrm{E}+02$ & $4.2 \mathrm{E}+02$ & $7.6 \mathrm{E}+01$ & $9.9 \mathrm{E}+02$ & 4.1E+02 & $1.9 \mathrm{E}+04$ & $9.1 \mathrm{E}+03$ \\
\hline MFO & $3.2 \mathrm{E}+10$ & $1.7 \mathrm{E}+05$ & $2.6 \mathrm{E}+03$ & $4.2 \mathrm{E}+02$ & $4.5 \mathrm{E}+01$ & $9.0 \mathrm{E}+02$ & $3.8 \mathrm{E}+02$ & $1.5 \mathrm{E}+04$ & $7.9 \mathrm{E}+03$ \\
\hline $\mathrm{ABC}$ & $9.2 \mathrm{E}+08$ & $6.6 \mathrm{E}+05$ & $1.2 \mathrm{E}+03$ & $5.0 \mathrm{E}+02$ & $3.0 \mathrm{E}+01$ & $5.7 \mathrm{E}+02$ & $5.0 \mathrm{E}+02$ & $3.0 \mathrm{E}+04$ & $1.5 \mathrm{E}+04$ \\
\hline FPA & $2.3 \mathrm{E}+11$ & $1.9 \mathrm{E}+08$ & $9.0 \mathrm{E}+04$ & $1.1 \mathrm{E}+03$ & $1.4 \mathrm{E}+02$ & $4.7 \mathrm{E}+03$ & $1.1 \mathrm{E}+03$ & $9.2 \mathrm{E}+04$ & $1.6 \mathrm{E}+04$ \\
\hline CS & $1.4 \mathrm{E}+05$ & $1.6 \mathrm{E}+05$ & $7.7 \mathrm{E}+01$ & $2.9 \mathrm{E}+02$ & $6.2 \mathrm{E}+01$ & $3.4 \mathrm{E}+02$ & $2.8 \mathrm{E}+02$ & $1.6 \mathrm{E}+04$ & $7.0 \mathrm{E}+03$ \\
\hline IWO & $6.9 \mathrm{E}+03$ & $2.6 \mathrm{E}+04$ & $1.2 \mathrm{E}+02$ & $7.4 \mathrm{E}+02$ & $7.8 \mathrm{E}+01$ & $3.5 \mathrm{E}+03$ & $7.2 \mathrm{E}+02$ & $2.0 \mathrm{E}+04$ & $7.7 \mathrm{E}+03$ \\
\hline $\mathrm{PSO}_{1}$ & $1.3 \mathrm{E}+09$ & $9.6 \mathrm{E}+03$ & $2.5 \mathrm{E}+02$ & $2.3 \mathrm{E}+02$ & $2.0 \mathrm{E}+01$ & $2.8 \mathrm{E}+02$ & $2.3 \mathrm{E}+02$ & $5.8 \mathrm{E}+03$ & $6.5 \mathrm{E}+03$ \\
\hline $\mathrm{PSO}_{2}$ & $1.2 \mathrm{E}+10$ & $5.8 \mathrm{E}+04$ & $9.3 \mathrm{E}+02$ & $2.0 \mathrm{E}+02$ & $1.2 \mathrm{E}+01$ & $2.7 \mathrm{E}+02$ & $2.0 \mathrm{E}+02$ & $3.6 \mathrm{E}+03$ & $6.1 \mathrm{E}+03$ \\
\hline HIDMS-PSO & $4.6 \mathrm{E}+03$ & $1.7 \mathrm{E}-03$ & $7.3 \mathrm{E}+01$ & $1.1 \mathrm{E}+02$ & 7.1E-02 & $1.8 \mathrm{E}+02$ & $1.1 \mathrm{E}+02$ & $4.2 \mathrm{E}+01$ & $5.5 \mathrm{E}+03$ \\
\hline *FFQ-HIDMS-PSO & $1.4 \mathrm{E}+03$ & $1.9 \mathrm{E}+03$ & $1.1 \mathrm{E}+02$ & $4.9 \mathrm{E}+01$ & $6.2 \mathrm{E}-01$ & $1.2 \mathrm{E}+02$ & 4. $5 \mathrm{E}+01$ & $3.2 \mathrm{E}+00$ & $5.3 \mathrm{E}+03$ \\
\hline
\end{tabular}

TABLE III

THE RESULTS OBTAINED FOR THE SECOND EXPERIMENT CONDUCTED USING THE CEC'05 TEST SUITE FOR PROBLEM SIZE OF 30 DIMENSIONS.

\begin{tabular}{|c|c|c|c|c|c|c|c|c|c|c|}
\hline & $\mathrm{F} 1$ & F2 & F3 & F4 & F5 & F6 & F7 & F8 & F9 & F10 \\
\hline HIDMS-PSO & $1.400 \mathrm{E}-12$ & $1.075 \mathrm{E}-03$ & $1.127 \mathrm{E}+06$ & $1.736 \mathrm{E}+03$ & $2.980 \mathrm{E}+03$ & $6.963 \mathrm{E}+01$ & $4.696 \mathrm{E}+03$ & $2.069 \mathrm{E}+01$ & $4.956 \mathrm{E}+01$ & $6.523 \mathrm{E}+01$ \\
\hline HPSO-TVAC & $5.495 \mathrm{E}-14$ & $4.782 \mathrm{E}-02$ & $1.745 \mathrm{E}+06$ & $2.997 \mathrm{E}+03$ & $5.459 \mathrm{E}+03$ & $1.092 \mathrm{E}+02$ & $4.696 \mathrm{E}+03$ & $2.099 \mathrm{E}+01$ & $3.638 \mathrm{E}+01$ & $9.984 \mathrm{E}+01$ \\
\hline FDR & $4.970 \mathrm{E}+02$ & $1.361 \mathrm{E}+03$ & $1.622 \mathrm{E}+07$ & $2.796 \mathrm{E}+03$ & $3.623 \mathrm{E}+03$ & $2.373 \mathrm{E}+06$ & $4.696 \mathrm{E}+03$ & $2.099 \mathrm{E}+01$ & $2.737 \mathrm{E}+02$ & $1.980 \mathrm{E}+02$ \\
\hline HCLDMS-PSO & $3.297 \mathrm{E}-12$ & $3.453 \mathrm{E}+01$ & $2.940 \mathrm{E}+06$ & $2.214 \mathrm{E}+03$ & $2.847 \mathrm{E}+03$ & $6.333 \mathrm{E}+01$ & $4.696 \mathrm{E}+03$ & $2.084 \mathrm{E}+01$ & $3.718 \mathrm{E}+01$ & $3.549 \mathrm{E}+01$ \\
\hline HCLPSO & $1.262 \mathrm{E}+01$ & $2.196 \mathrm{E}+01$ & $3.688 \mathrm{E}+06$ & $2.147 \mathrm{E}+03$ & $2.393 \mathrm{E}+03$ & $2.891 \mathrm{E}+05$ & $4.696 \mathrm{E}+03$ & $2.094 \mathrm{E}+01$ & $4.017 \mathrm{E}+00$ & $6.669 \mathrm{E}+01$ \\
\hline MNHPSO-JTVAC & $5.874 \mathrm{E}-14$ & $9.344 \mathrm{E}-03$ & $9.784 \mathrm{E}+05$ & $3.575 \mathrm{E}+03$ & $5.366 \mathrm{E}+03$ & $9.910 \mathrm{E}+01$ & $4.696 \mathrm{E}+03$ & $2.100 \mathrm{E}+01$ & $2.454 \mathrm{E}+01$ & $1.007 \mathrm{E}+02$ \\
\hline *FFQ-HIDMS-PSO & $1.177 \mathrm{E}-03$ & $7.955 \mathrm{E}+01$ & $3.440 \mathrm{E}+06$ & $3.175 \mathrm{E}+02$ & $1.267 \mathrm{E}+03$ & $1.670 \mathrm{E}+02$ & $4.696 \mathrm{E}+03$ & $2.091 \mathrm{E}+01$ & $2.109 \mathrm{E}+01$ & $3.091 \mathrm{E}+01$ \\
\hline
\end{tabular}

[2] Parsopoulos K.E. (2015) Particle Swarm Methods.In:Marti R., Panos P., Resende M. (eds) Handbook of Heuristics. Springer,Cham. https://doi.org/10.1007/978-3-319-07153-4_22-1

[3] Li, M., Liu, C., Li, K., Liao, X. and Li, K., 2020. Multi-task allocation with an optimized quantum particle swarm method. Applied Soft Computing, 96, p.106603.

[4] Santos Junior, J. and Silva do Monte Lima, J., 2018. Particle swarm optimization for 3D object tracking in RGB-D images. Computers and Graphics, 76, pp.167-180.

[5] F. T. Varna and P. Husbands, "HIDMS-PSO: A New Heterogeneous Improved Dynamic Multi-Swarm PSO Algorithm," 2020 IEEE Symposium Series on Computational Intelligence (SSCI), Canberra, Australia, 2020, pp. 473-480, doi: 10.1109/SSCI47803.2020.9308313.

[6] J.-M. Ame, J. Halloy, C. Rivault, C. Detrain, and J. L. Deneubourg, "Collegial decision making based on social amplification leads to optimal group formation.," Proc. Natl. Acad. Sci. U. S. A., vol. 103, no. 15, pp. 5835-40, Apr. 2006, doi: 10.1073/pnas.0507877103.

[7] X.-S. Yang, A new metaheuristic bat-inspired algorithm, Nature Inspired
Cooperative Strategies for Optimization (NICSO 2010), Springer, 2010.

[8] S. Mirjalili, S.M. Mirjalili, A. Lewis, Grey Wolf Optimizer, Adv. Eng. Software 69 (2014) 46-61.

[9] S. Arora, S. Singh, Butterfly optimization algorithm: a novel approach for global optimization, Soft Comput. 23 (3) (2018) 715-734.

[10] S. Mirjalili, A. Lewis, The Whale Optimization Algorithm, Adv. Eng. Software 95 (2016) 51-67.

[11] S. Mirjalili, Moth-flame optimization algorithm: A novel nature-inspired heuristic paradigm, Knowl.-Based Syst. 89 (2015) 228-249. M.A. Awadallah.

[12] D. Karaboga, B. Basturk, A powerful and efficient algorithm for numerical function optimization: artificial bee colony (ABC) algorithm, J. Global Optim. 39 (3) (2007) 459-471.

[13] A. R. Mehrabian and C. Lucas, "A novel numerical optimization algorithm inspired from weed colonization," Ecol. Inform., vol. 1, no. 4, pp. 355-366, Dec. 2006, doi:10.1016/j.ecoinf.2006.07.003.

[14] Yang XS. (2012) Flower Pollination Algorithm for Global Optimization. In: Durand-Lose J., Jonoska N. (eds) Unconventional Computation and 
TABLE IV

THE RESULTS OBTAINED FOR THE SECOND EXPERIMENT CONDUCTED USING THE CEC'05 TEST SUITE FOR PROBLEM SIZE OF 50 DIMENSIONS.

\begin{tabular}{|c|c|c|c|c|c|c|c|c|c|c|}
\hline & F1 & F2 & F3 & F4 & F5 & F6 & F7 & F8 & F9 & F10 \\
\hline HIDMS-PSO & $2.5 \mathrm{E}-09$ & $2.8 \mathrm{E}+01$ & $3.8 \mathrm{E}+06$ & $2.5 \mathrm{E}+04$ & $6.8 \mathrm{E}+03$ & $1.2 \mathrm{E}+02$ & $6.2 \mathrm{E}+03$ & $2.1 \mathrm{E}+01$ & $1.2 \mathrm{E}+02$ & $1.3 \mathrm{E}+02$ \\
\hline HPSO-TVAC & $1.0 \mathrm{E}-13$ & $1.9 \mathrm{E}+02$ & $4.4 \mathrm{E}+06$ & $3.1 \mathrm{E}+04$ & $1.6 \mathrm{E}+04$ & $1.7 \mathrm{E}+02$ & $6.2 \mathrm{E}+03$ & $2.1 \mathrm{E}+01$ & $1.1 \mathrm{E}+02$ & $1.9 \mathrm{E}+02$ \\
\hline FDR & $1.3 \mathrm{E}+03$ & $1.1 \mathrm{E}+04$ & 7.2E+07 & $2.6 \mathrm{E}+04$ & $8.2 \mathrm{E}+03$ & $9.9 \mathrm{E}+06$ & $6.2 \mathrm{E}+03$ & $2.1 \mathrm{E}+01$ & $5.6 \mathrm{E}+02$ & $4.3 \mathrm{E}+02$ \\
\hline HCLDMS-PSO & $6.9 \mathrm{E}-07$ & $2.8 \mathrm{E}+03$ & $1.1 \mathrm{E}+07$ & $2.2 \mathrm{E}+04$ & $7.5 \mathrm{E}+03$ & $2.4 \mathrm{E}+02$ & $6.2 \mathrm{E}+03$ & $2.1 \mathrm{E}+01$ & 1.1E+02 & $9.5 \mathrm{E}+01$ \\
\hline HCLPSO & $8.0 \mathrm{E}+00$ & $2.0 \mathrm{E}+03$ & $1.4 \mathrm{E}+07$ & $2.5 \mathrm{E}+04$ & $6.3 \mathrm{E}+03$ & $1.8 \mathrm{E}+05$ & $6.2 \mathrm{E}+03$ & $2.1 \mathrm{E}+01$ & $1.8 \mathrm{E}+01$ & $1.2 \mathrm{E}+02$ \\
\hline MNHPSO-JTVAC & $1.2 \mathrm{E}-13$ & $9.6 \mathrm{E}+01$ & $2.9 \mathrm{E}+06$ & $2.7 \mathrm{E}+04$ & $1.4 \mathrm{E}+04$ & $1.3 \mathrm{E}+02$ & $6.2 \mathrm{E}+03$ & $2.1 \mathrm{E}+01$ & $8.3 \mathrm{E}+01$ & $1.6 \mathrm{E}+02$ \\
\hline *FFQ-HIDMS-PSO & 4.3E- 02 & $1.8 \mathrm{E}+03$ & $1.2 \mathrm{E}+07$ & $5.3 \mathrm{E}+03$ & $3.5 \mathrm{E}+03$ & $4.0 \mathrm{E}+02$ & $6.2 \mathrm{E}+03$ & $2.1 \mathrm{E}+01$ & 4.4E+01 & $4.8 \mathrm{E}+01$ \\
\hline
\end{tabular}

TABLE V

THE RESULTS OBTAINED FOR THE THIRD EXPERIMENT CONDUCTED USING THE CEC'05 TEST SUITE FOR PROBLEM SIZE OF 30 DIMENSIONS.

\begin{tabular}{|c|c|c|c|c|c|c|c|c|c|c|}
\hline & $\overline{F 1}$ & $\bar{F} 2$ & F3 & F4 & F5 & F6 & F7 & F8 & F9 & F10 \\
\hline$\chi \mathrm{PSO}$ & $9.7 \mathrm{E}+00$ & $1.6 \mathrm{E}+01$ & $1.0 \mathrm{E}+07$ & $1.8 \mathrm{E}+03$ & $8.1 \mathrm{E}+03$ & $1.2 \mathrm{E}+03$ & $6.8 \mathrm{E}+03$ & $2.1 \mathrm{E}+01$ & $6.5 \mathrm{E}+01$ & $8.7 \mathrm{E}+01$ \\
\hline BBPSO & $0.0 \mathrm{E}+00$ & $9.3 \mathrm{E}-03$ & $1.3 \mathrm{E}+06$ & $2.3 \mathrm{E}+03$ & $5.3 \mathrm{E}+03$ & $2.8 \mathrm{E}+01$ & $4.7 \mathrm{E}+03$ & $2.1 \mathrm{E}+01$ & $5.6 \mathrm{E}+01$ & $7.6 \mathrm{E}+01$ \\
\hline DMSPSO & 3.1E+02 & $7.8 \mathrm{E}+02$ & $5.6 \mathrm{E}+06$ & $8.6 \mathrm{E}+02$ & $4.3 \mathrm{E}+03$ & $2.7 \mathrm{E}+07$ & $4.3 \mathrm{E}+03$ & $2.1 \mathrm{E}+01$ & $4.8 \mathrm{E}+01$ & $8.0 \mathrm{E}+01$ \\
\hline FIPS & $5.3 \mathrm{E}+02$ & $1.5 \mathrm{E}+04$ & $1.9 \mathrm{E}+07$ & $2.1 \mathrm{E}+04$ & $1.2 \mathrm{E}+04$ & $2.5 \mathrm{E}+07$ & $7.5 \mathrm{E}+03$ & $2.1 \mathrm{E}+01$ & $5.4 \mathrm{E}+01$ & $1.5 \mathrm{E}+02$ \\
\hline UPSO & $1.3 \mathrm{E}+03$ & $7.6 \mathrm{E}+03$ & $5.3 \mathrm{E}+07$ & $1.9 \mathrm{E}+04$ & $1.3 \mathrm{E}+04$ & $1.2 \mathrm{E}+07$ & $7.5 \mathrm{E}+03$ & $2.1 \mathrm{E}+01$ & $7.8 \mathrm{E}+01$ & $1.6 \mathrm{E}+02$ \\
\hline CLPSO & $0.0 \mathrm{E}+00$ & $3.8 \mathrm{E}+02$ & $1.2 \mathrm{E}+07$ & $5.4 \mathrm{E}+03$ & $4.0 \mathrm{E}+03$ & $1.8 \mathrm{E}+01$ & $4.7 \mathrm{E}+03$ & $2.1 \mathrm{E}+01$ & $0.0 \mathrm{E}+00$ & $8.0 \mathrm{E}+01$ \\
\hline${ }^{*}$ FFQ-HIDMS-PSO & $1.1 \mathrm{E}-03$ & $8.1 \mathrm{E}+01$ & $3.4 \mathrm{E}+06$ & $4.3 \mathrm{E}+02$ & $1.3 \mathrm{E}+03$ & $1.9 \mathrm{E}+02$ & $4.7 \mathrm{E}+03$ & $2.1 \mathrm{E}+01$ & $2.4 \mathrm{E}+01$ & $3.2 \mathrm{E}+01$ \\
\hline
\end{tabular}

TABLE VI

THE RESULTS OBTAINED FOR THE THIRD EXPERIMENT CONDUCTED USING THE CEC'05 TEST SUITE FOR PROBLEM SIZE OF 50 DIMENSIONS.

\begin{tabular}{|c|c|c|c|c|c|c|c|c|c|c|}
\hline & F1 & F2 & F3 & F4 & F5 & F6 & F7 & F8 & F9 & F10 \\
\hline$\chi \mathrm{PSO}$ & $9.7 \mathrm{E}+00$ & $7.8 \mathrm{E}+02$ & $2.0 \mathrm{E}+07$ & $2.8 \mathrm{E}+04$ & $1.1 \mathrm{E}+04$ & $6.4 \mathrm{E}+06$ & $6.2 \mathrm{E}+03$ & $2.1 \mathrm{E}+01$ & $1.8 \mathrm{E}+02$ & $1.8 \mathrm{E}+02$ \\
\hline BBPSO & $0.0 \mathrm{E}+00$ & $2.9 \mathrm{E}+02$ & $3.7 \mathrm{E}+06$ & $3.0 \mathrm{E}+04$ & $1.3 \mathrm{E}+04$ & $5.8 \mathrm{E}+01$ & $6.2 \mathrm{E}+03$ & $2.1 \mathrm{E}+01$ & $1.3 \mathrm{E}+02$ & $1.8 \mathrm{E}+02$ \\
\hline DMSPSO & $3.9 \mathrm{E}+02$ & $9.7 \mathrm{E}+02$ & $1.3 \mathrm{E}+07$ & $1.3 \mathrm{E}+04$ & $5.5 \mathrm{E}+03$ & $1.8 \mathrm{E}+07$ & $6.1 \mathrm{E}+03$ & 2.1E+01 & $9.9 \mathrm{E}+01$ & $1.7 \mathrm{E}+02$ \\
\hline FIPS & $1.7 \mathrm{E}+03$ & $2.6 \mathrm{E}+04$ & $5.9 \mathrm{E}+07$ & $3.4 \mathrm{E}+04$ & $1.6 \mathrm{E}+04$ & $8.0 \mathrm{E}+07$ & $1.0 \mathrm{E}+04$ & 2.1E+01 & $1.5 \mathrm{E}+02$ & $3.9 \mathrm{E}+02$ \\
\hline UPSO & $7.1 \mathrm{E}+02$ & $4.2 \mathrm{E}+03$ & $5.3 \mathrm{E}+07$ & $1.4 \mathrm{E}+04$ & $1.2 \mathrm{E}+04$ & $2.7 \mathrm{E}+06$ & $7.4 \mathrm{E}+03$ & $2.1 \mathrm{E}+01$ & $6.5 \mathrm{E}+01$ & $1.4 \mathrm{E}+02$ \\
\hline CLPSO & $0.0 \mathrm{E}+00$ & $1.0 \mathrm{E}+04$ & $4.9 \mathrm{E}+07$ & $3.4 \mathrm{E}+04$ & $9.7 \mathrm{E}+03$ & $8.7 \mathrm{E}+01$ & $6.2 \mathrm{E}+03$ & $2.1 \mathrm{E}+01$ & $0.0 \mathrm{E}+00$ & $2.2 \mathrm{E}+02$ \\
\hline *FFQ-HIDMS-PSO & $4.6 \mathrm{E}-02$ & $1.8 \mathrm{E}+03$ & $1.2 \mathrm{E}+07$ & $5.4 \mathrm{E}+03$ & $3.5 \mathrm{E}+03$ & $4.5 \mathrm{E}+02$ & $6.2 \mathrm{E}+03$ & $2.1 \mathrm{E}+01$ & $4.6 \mathrm{E}+01$ & $5.7 \mathrm{E}+01$ \\
\hline
\end{tabular}

TABLE VII

RANKS OF MEAN PERFORMANCE FOR THE FIRST EXPERIMENT.

\begin{tabular}{|ccccc|}
\hline Algorithm & Avg(30D) & Final(30D) & Avg(50D) & Final(50D) \\
\hline *FFQ-HIDMS-PSO & 1.90 & 1 & 1.45 & 1 \\
HIDMS-PSO & 2.45 & 2 & 2.17 & 2 \\
ABC & 2.93 & 3 & 8.45 & 10 \\
CS & 3.90 & 4 & 4.17 & 3 \\
GWO & 5.21 & 5 & 5.00 & 4 \\
MFO & 6.52 & 6 & 8.10 & 9 \\
IWO & 6.83 & 7 & 7.14 & 7 \\
WOA & 7.24 & 8 & 7.83 & 8 \\
BOA & 8.07 & 9 & 9.69 & 11 \\
BA & 10.07 & 10 & 12.03 & 12 \\
FPA & 10.93 & 11 & 12.93 & 13 \\
PSO & 12.00 & 12 & 5.48 & 5 \\
PSO $_{2}$ & 12.00 & 12 & 6.55 & 6 \\
\hline
\end{tabular}

TABLE VIII

RANKS OF MEAN PERFORMANCE FOR THE SECOND EXPERIMENT.

\begin{tabular}{|ccccc|}
\hline Algorithm & Avg(30D) & Final(30D) & Avg(50D) & Final(50D) \\
\hline *FFQ-HIDMS-PSO & 2.64 & 1 & 2.32 & 1 \\
HCLDMS-PSO & 2.84 & 2 & 3.44 & 3 \\
HIDMS-PSO & 2.92 & 3 & 2.88 & 2 \\
HCLPSO & 3.60 & 4 & 3.48 & 4 \\
MNHPSO-JTVAC & 4.32 & 5 & 4.04 & 5 \\
HPSO-TVAC & 4.36 & 6 & 4.56 & 6 \\
FDR & 6.24 & 7 & 6.32 & 7 \\
\hline
\end{tabular}

TABLE IX

RANKS OF MEAN PERFORMANCE FOR THE THIRD EXPERIMENT.

\begin{tabular}{|ccccc|}
\hline Algorithm & Avg(30D) & Final(30D) & Avg(50D) & Final(50D) \\
\hline *FFQ-HIDMS-PSO & 2.08 & 1 & 2.16 & 1 \\
CLPSO & 2.32 & 2 & 3.32 & 2 \\
BBPSO & 2.80 & 3 & 3.48 & 4 \\
XPSO & 3.76 & 4 & 4.24 & 6 \\
DMSPSO & 4.16 & 5 & 3.44 & 3 \\
FIPS & 5.84 & 6 & 6.40 & 7 \\
UPSO & 6.04 & 7 & 4.16 & 5 \\
\hline
\end{tabular}

Natural Computation. UCNC 2012. Lecture Notes in Computer Science, vol 7445. Springer, Berlin, Heidelberg. doi:10.1007/978-3-642-32894727.

[15] Yang, X. (2016). Nature-inspired optimization algorithms.: Elsevier, pp.256-258

[16] Clerc, M. and Kennedy, J., 2002. The particle swarm - explosion, stability, and convergence in a multidimensional complex space. IEEE
Transactions on Evolutionary Computation, 6(1), pp.58-73.

[17] Kennedy, J., n.d. Bare bones particle swarms. Proceedings of the 2003 IEEE Swarm Intelligence Symposium. SIS'03 (Cat. No.03EX706),

[18] J.-J. Liang, P.N. Suganthan, Dynamic multi-swarm particle swarm optimizer, in: Proceedings of the IEEE Swarm Intelligence Symposium, IEEE, 2005, pp. 124-129.

[19] Mendes, R., Kennedy, J. and Neves, J., 2004. The Fully Informed Particle Swarm: Simpler, Maybe Better. IEEE Transactions on Evolutionary Computation, 8(3), pp.204-210.

[20] K.E. Parsopoulos, M.N. Vrahatis, UPSO: a unified particle swarm optimization scheme, in: Lecture Series on Computer and Computational Sciences: Proc. of Int. Conf. of Computational Methods in Sciences and Engineering, 2004, pp. 868-873.

[21] Liang, J., Qin, A., Suganthan, P. and Baskar, S., 2006. Comprehensive learning particle swarm optimizer for global optimization of multimodal functions. IEEE Transactions on Evolutionary Computation, 10(3), pp.281-295.

[22] A. Ratnaweera, S.K. Halgamuge, H.C. Watson, Self-organizing hierarchical particle swarm optimizer with time-varying acceleration coefficients, IEEE Trans. Evol. Comput. 8 (3) (2004) 240-255.

[23] T. Peram, K. Veeramachaneni, C.K. Mohan, Fitness-distance-ratio based particle swarm optimization, in: Proceedings of the 2003 IEEE Swarm Intelligence Symposium, IEEE, 2003, pp. 174-181.

[24] Wang, S., Liu, G., Gao, M., Cao, S., Guo, A. and Wang, J., 2020. Heterogeneous comprehensive learning and dynamic multi-swarm particle swarm optimizer with two mutation operators. Information Sciences, 540, pp.175-201.

[25] N. Lynn, P.N. Suganthan, Heterogeneous comprehensive learning particle swarm optimization with enhanced exploration and exploitation, Swarm Evol. Comput. 24 (2015) 11-24.

[26] Ghasemi, M., Akbari, E., Zand, M., Hadipour, M., Ghavidel, S. and Li, L., 2019. An Efficient Modified HPSO-TVAC-Based Dynamic Economic Dispatch of Generating Units. Electric Power Components and Systems, 47(19-20), pp.1826-1840.

[27] M. G. Epitropakis, V. P. Plagianakos, and M. N. Vrahatis, "Evolving cognitive and social experience in Particle Swarm Optimization through Differential Evolution: A hybrid approach,” Inf. Sci. (Ny)., 2012, doi: 10.1016/j.ins.2012.05.017. 